\title{
Napsin A levels in epithelial lining fluid as a diagnostic biomarker of primary lung adenocarcinoma
}

\author{
Akifumi Uchida', Takuya Samukawa'1, Tomohiro Kumamoto', Masahiro Ohshige', Kazuhito Hatanaka², \\ Yoshihiro Nakamura ${ }^{3}$, Keiko Mizuno ${ }^{1}$, Ikkou Higashimoto', Masami Sato ${ }^{3}$ and Hiromasa Inoue ${ }^{1 *}$ (D)
}

\begin{abstract}
Background: It is crucial to develop novel diagnostic approaches for determining if peripheral lung nodules are malignant, as such nodules are frequently detected due to the increased use of chest computed tomography scans. To this end, we evaluated levels of napsin A in epithelial lining fluid (ELF), since napsin A has been reported to be an immunohistochemical biomarker for histological diagnosis of primary lung adenocarcinoma.

Methods: In consecutive patients with indeterminate peripheral lung nodules, ELF samples were obtained using a bronchoscopic microsampling (BMS) technique. The levels of napsin A and carcinoembryonic antigen (CEA) in ELF at the nodule site were compared with those at the contralateral site. A final diagnosis of primary lung adenocarcinoma was established by surgical resection.

Results: We performed BMS in 43 consecutive patients. Among patients with primary lung adenocarcinoma, the napsin A levels in ELF at the nodule site were markedly higher than those at the contralateral site, while there were no significant differences in CEA levels. Furthermore, in 18 patients who were undiagnosed by bronchoscopy and finally diagnosed by surgery, the napsin A levels in ELF at the nodule site were identically significantly higher than those at the contralateral site. In patients with non-adenocarcinoma, there were no differences in napsin A levels in ELF. The area under the receiver operator characteristic curve for identifying primary lung adenocarcinoma was 0.840 for napsin $A$ and 0.542 for CEA.
\end{abstract}

Conclusion: Evaluation of napsin A levels in ELF may be useful for distinguishing primary lung adenocarcinoma.

Keywords: Lung cancer diagnosis, Primary lung adenocarcinoma, Epithelial lining fluid, Biomarkers, Bronchoscopy

\section{Background}

Lung cancer is one of the most common malignant tumours and carries a high mortality rate [1]. The recent increased use of chest computed tomography (CT) has resulted in more frequent incidental detection of peripheral lung nodules, and the most common malignant finding is primary lung adenocarcinoma [2,3]. Some novel tumour markers and imaging tests are useful for lung cancer [4], but these markers have inadequate diagnostic accuracy. Various circulating tumour markers for primary lung adenocarcinoma

\footnotetext{
* Correspondence: inoue-pulm@umin.net

'Department of Pulmonary Medicine, Graduate School of Medical and Dental Sciences, Kagoshima University, 8-35-1 Sakuragaoka, Kagoshima 890-8520, Japan

Full list of author information is available at the end of the article
}

have been identified. Carcinoembryonic antigen (CEA) and sialyl Lewis Xi antigen (SLX) are clinically established and useful for the management of primary lung adenocarcinoma. However, their values in serum are not suitable for screening or diagnosis of early-stage primary lung adenocarcinoma due to low sensitivity and specificity [5-7].

The pathological diagnosis of primary lung adenocarcinoma is usually based on tissue or cytological samples obtained by bronchoscopy. However, collection of such samples is sometimes difficult due to the location and size of the nodule or the condition of the patient $[2,8]$. CTguided needle biopsy and thoracoscopic lung biopsy are useful alternative methods, although the invasiveness of both procedures is a disadvantage $[9,10]$. Therefore, it is crucial to develop novel diagnostic tools, including other 
biomarkers and techniques for sample collection, for the accurate diagnosis of primary lung adenocarcinoma.

Bronchoscopic microsampling (BMS) has attracted attention as a new diagnostic tool for lung cancer (Fig. 1) [11]. With the BMS technique it is possible to obtain epithelial lining fluid (ELF), the liquid which covers the bronchial wall and alveolus, without the need for saline injection [12]. ELF is transported toward the trachea by ciliary movement, and for diagnostic purposes it is not necessary for ELF to contact the tumour directly. Biochemical substances, including biochemical markers, tumour makers, tumour-derived nucleic acids and drug concentrations, can be measured by analysing ELF without the invasiveness or sample dilution required when testing bronchoalveolar lavage fluid [11, 13-19]. Of note, a previous study reported that measurement of CEA and cytokeratin fragment 19 in ELF was a beneficial diagnostic adjunct in patients with a small peripheral lung nodule [11].

Napsin A, an aspartic protease, is mainly expressed in alveolar type II cells. Previous studies have shown that napsin A is present and active in the alveolar space [20-22]. Immunohistochemical reactivity for napsin A is positive in most cases of primary lung adenocarcinoma, but negative in most cases of squamous cell carcinoma as well as adenocarcinoma of other organs. Its local expression was reported to be useful for identifying the lung origin of metastatic adenocarcinoma [23, 24]. In comparison with napsin A, CEA showed less sensitivity and specificity for histologically diagnosing lung adenocarcinoma using immunohistochemical biomarkers [25].

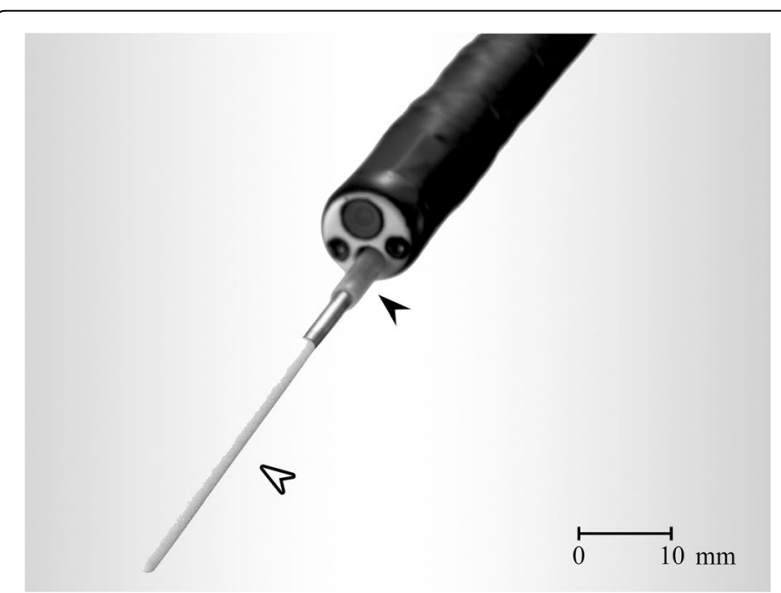

Fig. 1 Bronchoscopic microsampling (BMS) procedure. The BMS probe consists of a polyethylene outer sheath (black arrowhead) and an inner cotton fiber rod probe attached to a 30-mm-long stainless steel guide wire (white arrowhead). The probe is guided to the affected lesion in the subsegmental bronchus thorough the bronchoscope channel and the inner fiber rod probe (white arrowhead) is placed on the bronchial membrane as near as possible to the lung nodule. BMS:

bronchoscopic maicrosampling
Based on these findings, we hypothesised that napsin A levels in ELF at the primary lung adenocarcinoma site would be increased. To test this hypothesis, we compared napsin A levels in ELF at the malignant nodule site with those at the unaffected contralateral site. Furthermore, we measured CEA levels in ELF and compared the diagnostic usefulness of ELF-derived napsin A and CEA.

\section{Methods}

Study design

Patients with an indeterminate peripheral lung nodule who had bronchoscopy at Kagoshima University Medical and Dental Hospital from December 2012 to September 2016 were enroled in this study. We planned to analyse subjects who required surgical resection of the primary lung cancer. The selection criterion for the present study was the presence of a peripheral lung nodule with a diameter of less than $30 \mathrm{~mm}$ that was anticipated to be resectable with indications for surgery via bronchoscopy. Written informed consent for this study was obtained before bronchoscopy. This study was approved by the institutional review board of Kagoshima University Medical and Dental Hospital and the committee's reference number was 24-71. A final definitive histological diagnosis of primary lung adenocarcinoma was made by surgical resection in all patients. For serum marker analysis, healthy volunteers with normal chest radiographs and who had provided written informed consent were matched for gender and age and enrolled in the study as a control group.

\section{Microsampling probe and procedure}

The BMS probe used was the BC-401C (Fig. 1, Olympus Co., Tokyo, Japan). ELF samples were obtained both from a subsegmental bronchus as near as possible to the nodule, using fluoroscopy, and from the contralateral subsegmental bronchus using first the BMS technique followed by endobronchial ultrasound sonography and fluoroscopy with subsequent transbronchial lung biopsy. The BMS method was performed as previously described [12]. After inserting a bronchoscope, the BMS probe in the outer sheath was inserted through the bronchoscope and the inner probe was advanced from the outer sheath toward the affected lesion at the subsegmental bronchus, and then the inner probe was placed on the bronchial membrane for $10 \mathrm{~s}$ to absorb the ELF. The inner probe was then immediately withdrawn into the outer sheath. This procedure was repeated three times. We also obtained ELF from the corresponding subsegmental bronchus of the contralateral lung as an internal control. After the BMS procedures, the inner probe tips were frozen at $-80{ }^{\circ} \mathrm{C}$. They were weighed and then ELF was extracted by stirring for $1 \mathrm{~min}$ 
following the addition of $3 \mathrm{~mL}$ of saline. The tips were then dried and weighed again to measure the ELF volume and calculate the dilution factor.

\section{Measurement}

The napsin A levels in both ELF (ELF-napsin A) and serum (serum-napsin A) were measured by enzymelinked immunosorbent assay with the Human Napsin A Assay Kit (Immuno-Biological Laboratories Co., Gunma, Japan). The CEA levels in ELF (ELF-CEA) were determined by chemiluminescent enzyme immune assay with Lumipulse Presto CEA (Fujirebio Inc., Saitama, Japan), and those in serum (serum-CEA) were quantified by electro-chemiluminescence immune assay with Cobas 800 (Roche Diagnostics K.K., Tokyo, Japan). Both ELFnapsin A and ELF-CEA were expressed per unit volume after correction for the dilution factor.

\section{Statistical analysis}

The patients who were diagnosed as other than primary lung adenocarcinoma were treated as non-adenocarcinoma. Data were analysed using SPSS version 23 software (IBM SPSS, Chicago, USA). Differences in serum-napsin A and serum-CEA between patients with primary lung adenocarcinoma and those in the control group were analysed by the Mann-Whitney $U$ test. Differences in the variables related to ELF-napsin A and ELF-CEA between the nodule site and contralateral site in the patients who underwent bronchoscopy were compared by the nonparametric Wilcoxon signed-rank test, since the data were not normally distributed. Differences in ELF-napsin A at the nodule site between the patients with primary lung adenocarcinoma and those with non-adenocarcinoma were analysed by the Mann-Whitney $U$ test. The data for each histological subtype of primary lung adenocarcinoma were compared using the nonparametric Kruskal-Wallis analysis of variance test. Correlations were examined using Spearman's correlation test. Statistical significance was defined as $P<0.05$. Receiver operating characteristic (ROC) curve analysis was used to assess the ability of ELF-napsin A and ELF-CEA, respectively, to predict the diagnosis of primary lung adenocarcinoma. In ROC analyses, the ELF values at the contralateral site in patients with primary lung adenocarcinoma and at the bilateral sites in patients with non-adenocarcinoma were treated as negative controls. The difference between the areas under the ROC curves (AUCs) for ELF-napsin A and ELF-CEA were performed with EZR version 1.36 (Saitama Medical Center, Jichi Medical University, Saitama, Japan), which is a graphical user interface for $\mathrm{R}$ (The $\mathrm{R}$ Foundation for Statistical Computing, Vienna, Austria). Any samples with values below the lower limit of quantification were assigned a value of half of the lower quantification limit for analysis.

\section{Result}

\section{Patients characteristics}

We performed BMS in 43 consecutive patients according to our selection criteria. Of 43 patients, 3 patients could not have a surgery because of distant metastasis. Five patients refused surgery for definitive diagnosis. Finally, total 8 patients were excluded, the remaining 35 patients were included in the analysis.

All patients underwent BMS followed by surgical resection without complications, except for mycobacterium infection, allowing for the determination of histological features. Of 35 patients, 27 patients were diagnosed with primary lung adenocarcinoma, and two patients were diagnosed as primary lung adenosquamous carcinoma and combined large cell neuroendocrine carcinoma (LCNEC); LCNEC with adenocarcinoma, respectively. In patients with non-adenocarcinoma, two patients were diagnosed with primary lung squamous cell carcinoma, one patient was diagnosed as primary lung large cell carcinoma, and one patient was diagnosed with metastatic carcinoma from nasopharyngeal carcinoma. The other two patients were identified as mycobacterium tuberculosis and nontuberculous mycobacterial infection, respectively (Table 1).

Patients with primary adenocarcinoma were 48 to 80 years of age, with a median age of 67 years. In 11 patients, the diagnosis of primary lung adenocarcinoma was obtained by transbronchial lung biopsy or curettage, while the other 18 patients were diagnosed by surgical resection. The pathological stage was determined surgically to be stage IA in 25 patients, stage IB in three, and stage IIIA in one. The diameter of the primary nodule at surgery was $22(9-36) \mathrm{mm}$, median (range). One representative case is shown in Fig. 2. This partly solid nodule with a diameter of $20 \mathrm{~mm}$ was seen in the right upper lobe on CT. It was not diagnosed by bronchoscopy. ELF-napsin A at the nodule site was $20911 \mathrm{ng} \cdot \mathrm{mL}^{-1}$, and that at the contralateral site was $370 \mathrm{ng} \cdot \mathrm{mL}^{-1}$. In this case, a final diagnosis of primary lung adenocarcinoma was established by surgical resection and napsin A and CEA were strongly expressed in adenocarcinoma cells on immunohistological examination.

\section{Biological marker levels in ELF and serum}

Among the patients with primary lung adenocarcinoma, ELF-napsin A at the nodule site was 11091 (326-55729) $\mathrm{ng} \cdot \mathrm{mL}^{-1}$ after correction for the dilution factor, while that at the contralateral site was $805(180-5266) \mathrm{ng} \cdot \mathrm{mL}^{-1}$, indicating a significant difference $(P<0.001$, Fig. 3a). Conversely, ELF-CEA at the nodule site was 135 (6-7799) ng.mL ${ }^{-1}$, and that at the contralateral site was $158(6-1806)$ ng. $\mathrm{mL}^{-1}$, indicating no significant difference $(P=0.634$, Fig. $3 \mathrm{~b})$. Furthermore, in 18 patients who were undiagnosed by bronchoscopy and finally diagnosed by surgery, ELF-napsin 
Table 1 Clinical characteristics of patient cohort

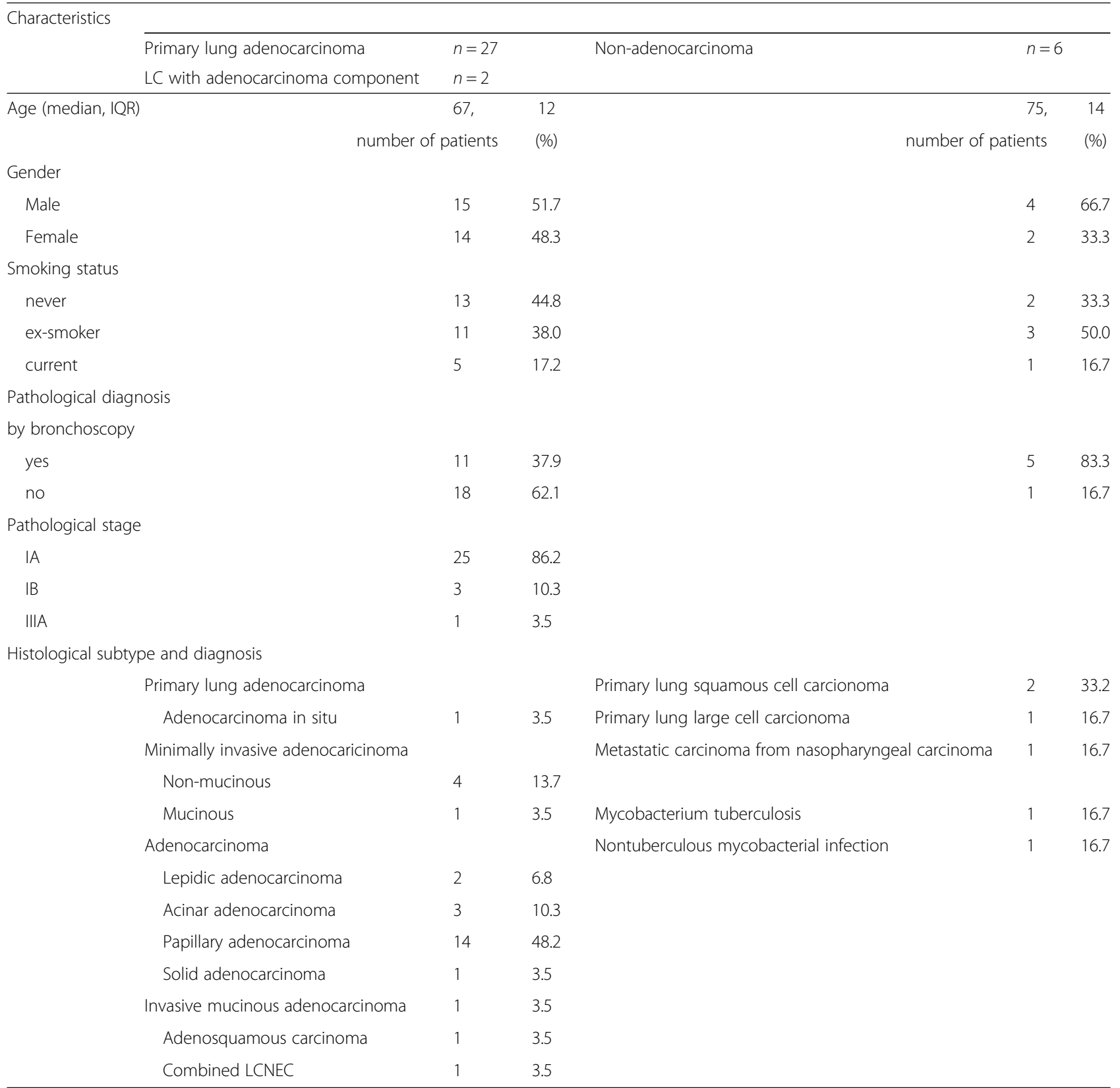

LC Lung carcinoma, IQR Interquartile range, LCNEC Large cell neuroendocrine carcinoma

Data are presented as number or \%, unless otherwise stated

Pathological stage is classified according to the TNM Classification of Malignancy Tumors, 7th Edition

Histological type is categorised according to the WHO Classification of Tumors of the Lung, Pleura, Thymus and Heart, Fourth Edition

A at the nodule site was $8438(326-52337) \mathrm{ng} \cdot \mathrm{mL}^{-1}$, and that at the contralateral site was $704(235-5266) \mathrm{ng} \cdot \mathrm{mL}^{-1}$. In this population there were identically significant differences between ELF-napsin A at the nodule site and those at the contralateral site $(P=0.001$, Fig. $3 c)$, as well as no difference between ELF-CEA at the nodule site, 135 (6-545) $\mathrm{ng} \cdot \mathrm{mL}^{-1}$, and that at the contralateral site, $201(6-1003)$ ng. $\mathrm{mL}^{-1}(P=0.571$, Fig. 3d).
In serum analysis, serum-napsin $\mathrm{A}$ in patients with primary lung adenocarcinoma was $16.1(2.0-366.1) \mathrm{ng} \cdot \mathrm{mL}^{-1}$, and that in the control group was $23.3(3.1-219.5) \mathrm{ng} \cdot \mathrm{mL}$ ${ }^{-1}$, indicating no significant difference $(P=0.251)$. Similarly, there was no difference between serum-CEA in patients with primary lung adenocarcinoma, 3.3 (0.4-15.7) ng. $\mathrm{mL}^{-1}$, and that in the control group, $1.8(0.6-9.1)$ ng.mL $L^{-1},(P=0.056)$. 


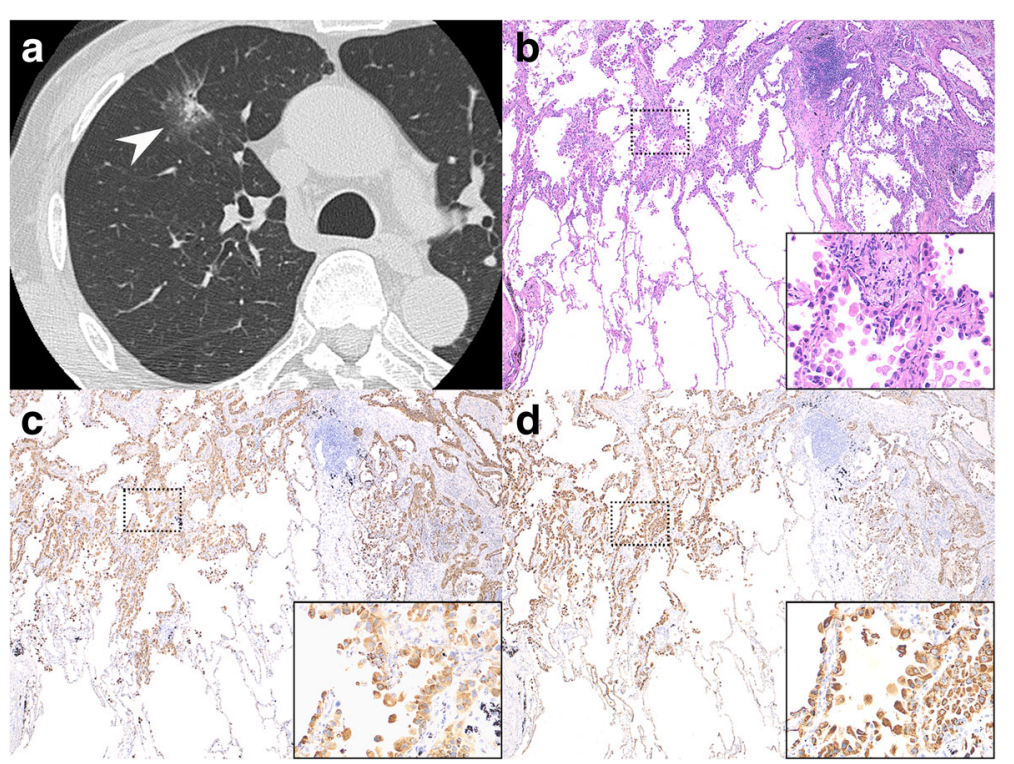

Fig. 2 Computed tomography (CT) and immunohistological findings in a representative case. a A lung CT image shows a 20-mm, partly solid, ground-glass opacity nodule (arrowhead) in the right upper lobe. b Minimally invasive adenocarcinoma. The cancer demonstrates predominant lepidic growth with $<5$-mm invasion. The cancer cells have polygonal cytoplasm and atypical nuclei (inset) (hematoxylin-eosin staining, original magnification $\times 40$ and high magnification $\times 100)$. c Immunohistological staining for napsin A. Napsin A is strongly positive in adenocarcinoma cells (inset for greater detail) (original magnification $\times 40$ and high magnification $\times 100$ ). $\mathbf{d}$ Immunohistological staining for CEA. CEA is strongly positive in adenocarcinoma cells (inset for greater detail) (original magnification $\times 40$ and high magnification $\times 100$ ). CT: computed tomography; CEA: carcinoembryonic antigen

In patients with non-adenocarcinoma, ELF-napsin $\mathrm{A}$ at the nodule site was 2041 (398-5108) ng.mL ${ }^{-1}$, and that at the contralateral site was $1719(425-4186) \mathrm{ng} \cdot \mathrm{mL}$ ${ }^{-1}$, indicating no significant difference $(P=0.463$, Fig. $4 a)$. ELF-napsin A at the nodule site was significantly higher in primary lung adenocarcinoma than in nonadenocarcinoma ( $P=0.024$, Fig. $4 b)$.

There was no significant difference in ELF-napsin A or ELF-CEA between histological subtypes of primary lung adenocarcinoma $(P=0.919$ and $P=0.590$, respectively). There was no significant difference in ELF-napsin A or ELF-CEA between smoking history and pathological stage (data not shown).

\section{Relationship between ELF and other clinical parameters}

There was no significant correlation between ELF-napsin A and serum-napsin A $(P=0.916, r=0.021$, Fig. 5a). There was no significant correlation between ELF napsin A and tumour sizes at surgery, however ELF-napsin A tended to rise as tumour sizes increased ( $P=0.053, r=0.362$, Fig. $5 b)$.

\section{Diagnostic values of napsin A levels in ELF}

We used ROC curve analysis to evaluate the sensitivity and specificity of ELF-napsin A and ELF-CEA as biomarkers for distinguishing primary lung adenocarcinoma (resected from 29 nodule sites) from normal tissue and non-adenocarcinoma (resected from 29 contralateral sites in primary lung adenocarcinoma and 12 bilateral sites in non-adenocarcinoma) (Fig. 6). The AUCs for nodule sites versus negative controls were 0.840 for ELF-napsin A and 0.542 for ELF-CEA (Table 2). The AUC of ELF-napsin A was significantly higher than that of ELF-CEA $(P<0.001)$. It was not possible to perform an ROC analysis for ELF-napsin A in combination with ELF-CEA using a binary logistic regression model, because the $p$-value of an ROC curve analysis for ELF-CEA was not significant. The optimal cut-off values for predicting the diagnosis of primary lung adenocarcinoma were $3280 \mathrm{ng} \cdot \mathrm{mL}^{-1}$ for ELF-napsin A, with a sensitivity and specificity of $79.3 \%$ and $82.9 \%$, respectively, and $82 \mathrm{ng} \cdot \mathrm{mL}^{-1}$ for ELF-CEA, with sensitivity and specificity values of $75.9 \%$ and $34.1 \%$, respectively (Table 2 ).

\section{Discussion}

In this study, we demonstrated that ELF-napsin A was a useful diagnostic marker for primary lung adenocarcinoma. ELF-napsin A at the nodule site was significantly higher in patients with primary lung adenocarcinoma. Moreover, except for three patients, ELF-napsin A at the nodule site was higher than that at the contralateral site. In the representative case shown in Fig. 2, many partly solid nodules could not be recognized on fluoroscopy or ultrasound sonography and were undiagnosed by bronchoscopy. Even in such patients, however, ELF-napsin A was significantly elevated. These findings suggested that in patients with elevated ELF-napsin A and no histological diagnosis of 

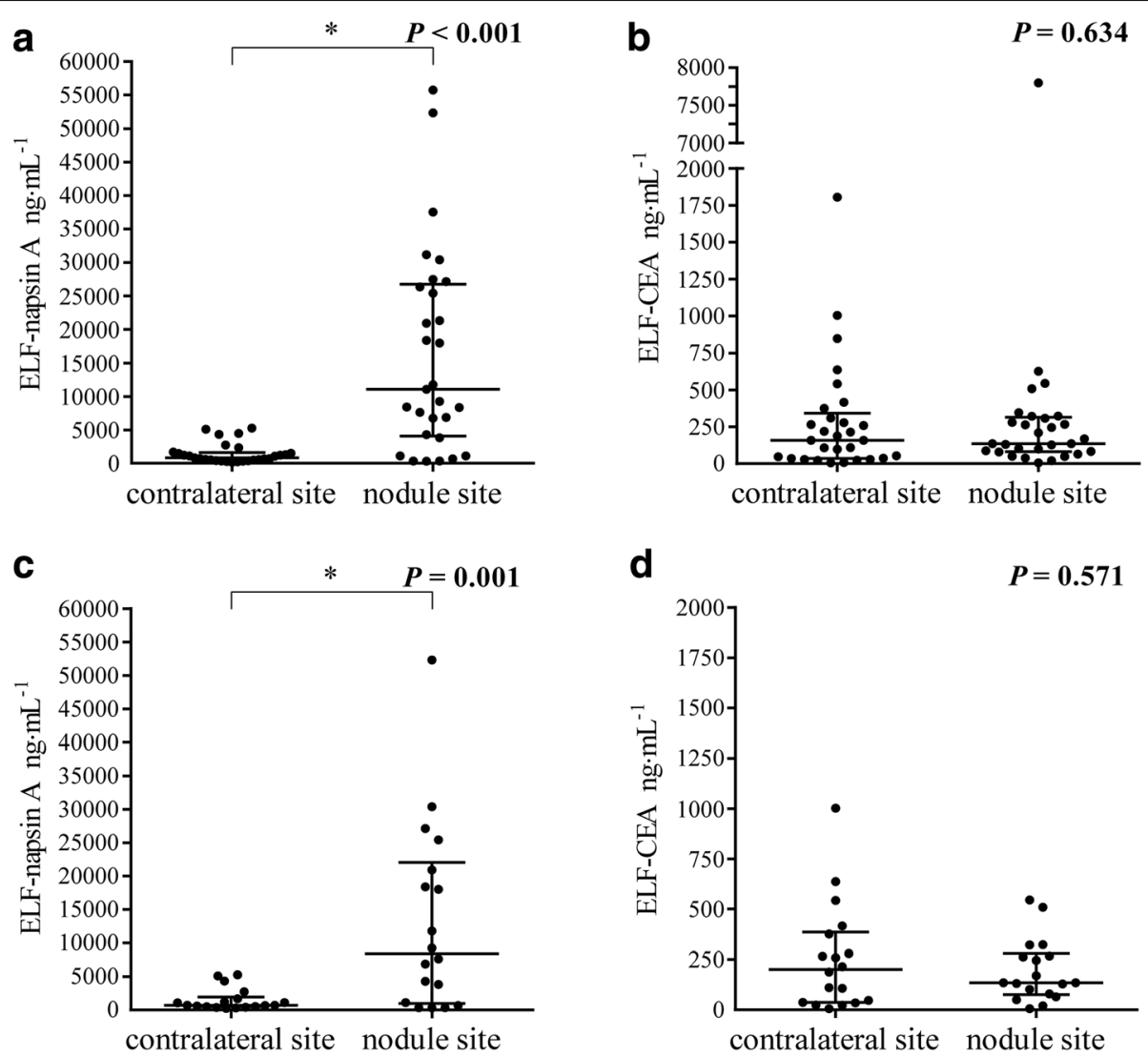

Fig. 3 Values of napsin A and CEA in ELF in the patients with primary lung adenocarcinoma. a Levels of napsin A in ELF (ELF-napsin A) in the patients with primary lung adenocarcinoma $(n=29, P<0.001)$. $\mathbf{b}$ Values of CEA in ELF (ELF-CEA) in the patients with primary lung adenocarcinoma $(n=29, P=0.634)$. c ELF-napsin $A$ in the patients with primary lung adenocarcinoma who were undiagnosed by bronchoscopy and finally diagnosed by surgery $(n=18, P=0.001)$. $\mathbf{d}$ ELF-CEA in the patients with primary lung adenocarcinoma who were undiagnosed by bronchoscopy and finally diagnosed by surgery $(n=18, P=0.571)$. CEA: carcinoembryonic antigen; ELF: epithelial lining fluid
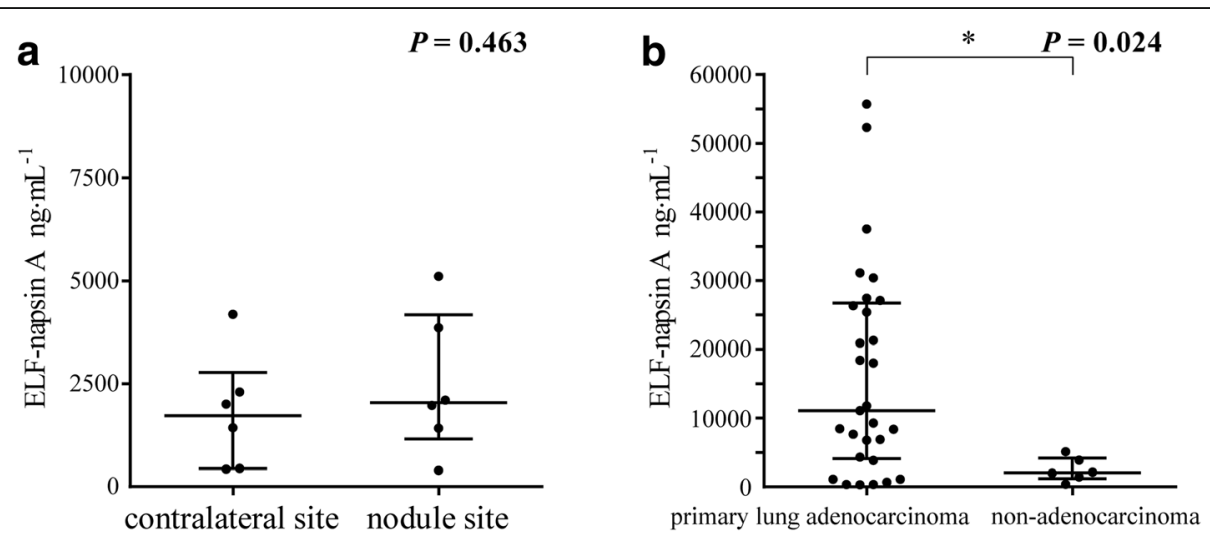

Fig. 4 Napsin A levels in ELF in patients with primary lung adenocarcinoma and those with non-adenocarcinoma. a Values of napsin A in ELF (ELF-napsin A) in the patients with non-adenocarcinoma $(n=6, P=0.463)$. b ELF-napsin A at the nodule site in primary lung adenocarcinoma $(n=29)$ and in non-adenocarcinoma $(n=6)(P=0.024)$. ELF: epithelial lining fluid 

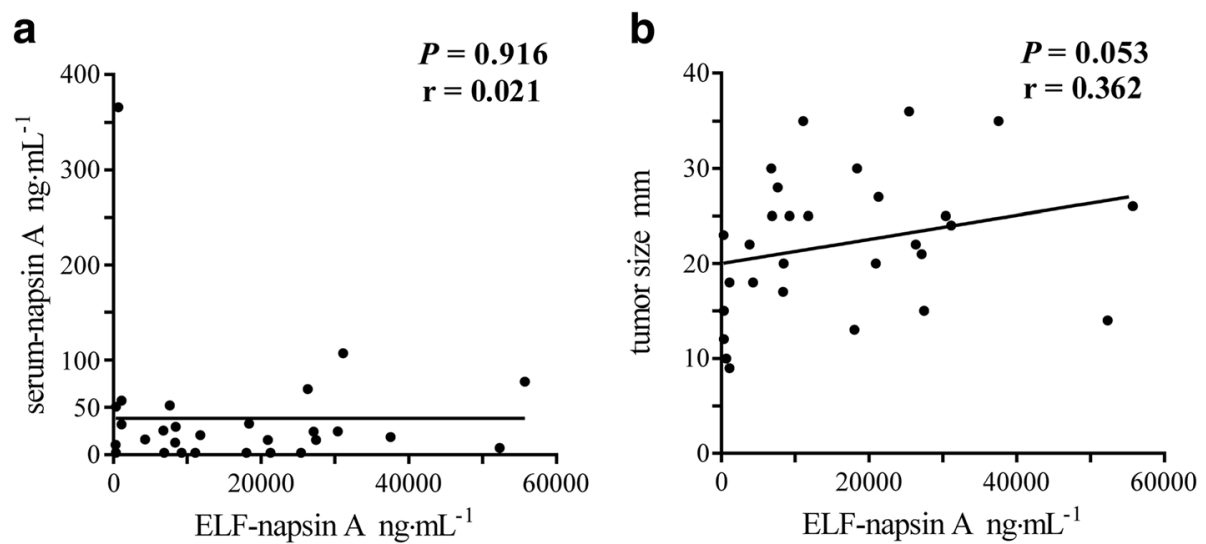

Fig. 5 Correlations of levels of napsin A in ELF. a Correlation between the levels of napsin A in ELF (ELF-napsin A) and the serum levels of napsin A (serum-napsin A) $(n=28, P=0.916, r=0.021)$. b Correlation between ELF-napsin A and the tumour size at surgery $(n=29, P=0.053, r=0.362)$. ELF: epithelial lining fluid

primary lung carcinoma by bronchoscopy, it is advisable to perform further evaluation with a high suspicion of primary lung adenocarcinoma. Furthermore, in ROC curve analysis, the AUC for ELF-napsin A was clearly higher than that for ELF-CEA, proving that ELF-napsin A is more valuable for diagnosing primary lung adenocarcinoma.

Because of the small diameters and complicated divergence patterns of bronchi, the detection rate of small lung nodules by bronchoscopy is not high. While the use of fluoroscopy, ultrasound sonography, and new bronchoscopy devices has gradually increased the rate of successful bronchoscopic diagnosis [8, 26-28], it is still not sufficient. If a definite diagnosis is not obtained by

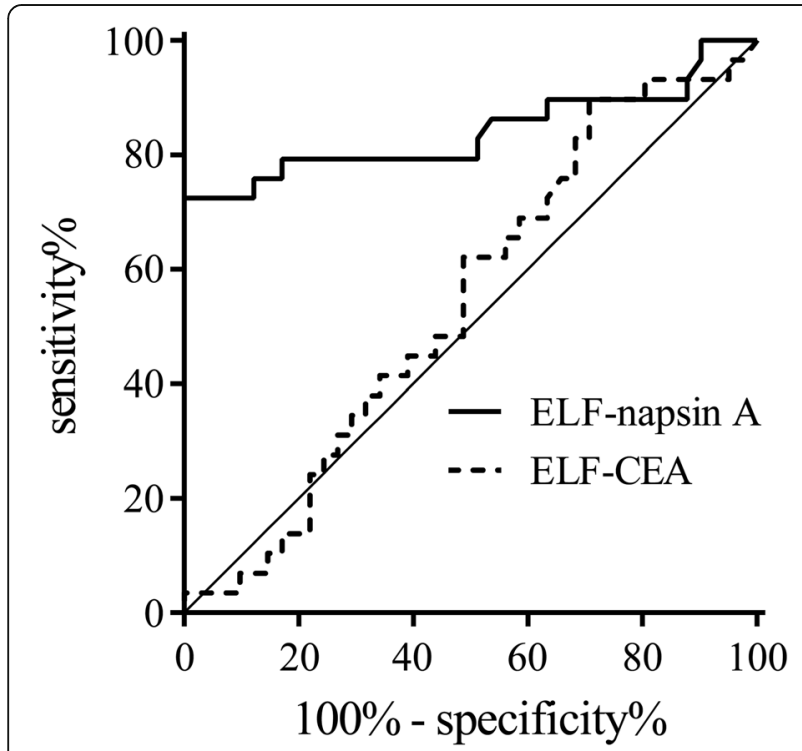

Fig. 6 Receiver operating characteristic (ROC) curve for napsin A and CEA in ELF. The ROC curves for napsin A in ELF (ELF-napsin A, solid line) and CEA in ELF (ELF-CEA, dashed line). The areas under the ROC curves are 0.840 for ELF-napsin A and 0.542 for ELF-CEA bronchoscopy, CT-guided needle biopsy or thoracoscopic lung biopsy are necessary, but both procedures are highly invasive and carry risks of grave complications $[9,10]$. It is desirable to develop a diagnostic tool with fewer complications, more convenience, and higher sensitivity and specificity.

The BMS technique, used to obtain ELF with bronchoscopy, is less invasive than bronchoalveolar lavage or transbronchial biopsy. Tumour-derived proteins and nucleic acids are contained in ELF close to a nodule, and the ELF is then transported toward the central bronchi by ciliary movement. As a result, the characteristics of a nodule can be evaluated by investigating ELF and direct access to the nodule is not necessary [11, 17-19]. A previous report found that the measurement of three biomarkers in ELF, namely CEA, cytokeratin fragment 19 and SLX, were useful for diagnosis of a small lung nodule [11]. But since these tumour markers are not specific for primary lung carcinoma, their utility for distinguishing primary lung carcinoma from metastatic cancer is uncertain. In contrast, napsin A is used as a diagnostic biomarker in immunohistochemical analyses for the purpose of discriminating between primary and metastatic lung carcinoma [23, 24].

In the present study, we demonstrated that ELF-napsin $\mathrm{A}$ at nodule sites was significantly higher than that at unaffected contralateral sites. The mechanism underlying this elevation in patients with primary lung adenocarcinoma is not known for certain, but it is probably due to overexpression of napsin A on primary lung adenocarcinoma tissue [29] in the alveolar space [20-22]. It may be that napsin A expressed by primary lung adenocarcinoma is released into the alveolar space and transported toward the central bronchi. The molecular weight of napsin $\mathrm{A}$ is approximately $38 \mathrm{kDa}$ [30], while that of CEA is estimated to be $180 \mathrm{kDa}$ [31]. This difference may account for the 
Table 2 ROC analysis comparing ELF-napsin A and ELF-CEA

\begin{tabular}{llllllllll}
\hline & AUC & $95 \% \mathrm{Cl}, \%$ & Cut off point & Sensitivity, \% & Specificity, \% & PPV, \% & NPV, \% & Positive LR & Negative LR \\
\hline ELF-napsin A & 0.840 & 72.8 to 95.3 & $3280 \mathrm{ng} \cdot \mathrm{mL}^{-1}$ & 79.3 & 82.9 & 76.7 & 85.0 & 4.64 & 0.25 \\
ELF-CEA & 0.542 & 40.7 to 67.8 & $82 \mathrm{ng} \cdot \mathrm{mL}^{-1}$ & 75.9 & 34.1 & 44.9 & 66.7 & 1.15 & 0.71
\end{tabular}

ELF Epithelial lining fluid, CEA Carcinoembryonic antigen, AUC Area under the ROC curve, CI Confidence interval, PPV Positive predictive value, NPV Negative predictive value, $L R$ Likehood ratio

discrepancy in the detected levels of these markers in ELF. Furthermore, ELF-napsin A at nodule sites tended to rise according to increase in tumour size at surgery, suggesting that the expression of napsin A was greater with increased mass and secretory ability of primary lung adenocarcinoma, regardless of its histological subtype.

Serum-napsin A in patients with primary lung adenocarcinoma was not elevated compared with that in the control group. These results were consistent with our previous findings [32]. CEA is a commonly recommended biomarker in the management of lung cancer [33,34]. But, because of its low sensitivity, it has not been generally recommended as a tool for the early screening of lung carcinoma, and it has been used as a potential prognostic biomarker and not a diagnostic biomarker. Moreover, CEA is not in itself a sufficiently strong indicator to guide treatment decisions for lung cancer [35]. A combination of biomarkers identifying primary lung adenocarcinoma may be helpful in distinguishing early-stage lung adenocarcinoma from benign lung disease, which presents as suspicious lung nodules.

The current study had several limitations. First, our sample size was small. Second, we could determine ELF-napsin $\mathrm{A}$ and ELF-CEA in cases of non-adenocarcinoma, including benign tumours and inflammation, in only a few patients. Last, the median diameter of primary lung adenocarcinoma at surgery was $22 \mathrm{~mm}$. Therefore, our results should be confirmed in a larger cohort study, which includes various diseases and smaller-size lung nodules.

\section{Conclusion}

In summary, elevated napsin A levels in ELF were found in patients with primary lung adenocarcinoma, and the levels tended to rise as tumour size increased at surgery. Our findings suggest that napsin A levels in ELF may be clinically useful for distinguishing primary lung adenocarcinoma.

\section{Abbreviations \\ AUC: Areas under the ROC curve; BMS: Bronchoscopic microsampling; CEA: Carcinoembryonic antigen; CT: Computed tomography; ELF: Epithelial lining fluid; ELF-CEA: CEA levels in ELF; ELF-napsin A: Napsin A levels in ELF; ROC: Receiver operating characteristic; serum-CEA: CEA levels in serum; serum-napsin A: Napsin A levels in serum; SLX: Sialyl Lewis Xi antigen}

\section{Acknowledgements}

We thank Asami Harumatsu, Department of Pulmonary Medicine, Graduate School of Medical and Dental Sciences, Kagoshima University for their assistance in data collection.
Funding

This study was supported in part by Grants-in-Aid for Scientific Research from the Japan Society for the Promotion of Science (JSPS, KAKENHI grant 15 K09179).

\section{Availability of data and materials}

The datasets generated and analysed this study are not publicly available due to confidentiality of patients and participants. However, the data are available from the corresponding author on reasonable request.

\section{Authors' contributions}

$\mathrm{AU}, \mathrm{TS}, \mathrm{MO}$ and $\mathrm{HI}$ designed this study. $\mathrm{AU}, \mathrm{TK}$ and $\mathrm{MO}$ contribute to the acquisition and analysis of data. AU, TS, KM, IH and $\mathrm{HI}$ contributed the data interpretation. $\mathrm{KH}, \mathrm{YN}$ and MS revised this manuscript critically for important intellectual content. All authors read and approved the final manuscript.

\section{Ethics approval and consent to participate}

This study was approved by the institutional review board of Kagoshima University Medical and Dental Hospital and the committee's reference number was 24-71. Written informed consent for this study was obtained before bronchoscopy.

\section{Consent for publication}

Witten informed consent for publication of all images in this manuscript was obtained from the patients.

\section{Competing interests}

Hiromasa Inoue reports grants from Astellas, AstraZeneca, BoehringerIngelheim, ChugaiPharm, GlaxoSmithKline, Pfizer, MerckSharp\&Dohme, TeijinPharma, Torii, personal fees from Astellas, AstraZeneca, Boehringer-Ingelheim, Chugai-Pharm, GlaxoSmithKline, Kyorin, MerckSharp\&Dohme, MeijiSeikaPharma, Novartis, Otsuka, Pfizer, outside the submitted work.

\section{Publisher's Note}

Springer Nature remains neutral with regard to jurisdictional claims in published maps and institutional affiliations.

\section{Author details}

${ }^{1}$ Department of Pulmonary Medicine, Graduate School of Medical and Dental Sciences, Kagoshima University, 8-35-1 Sakuragaoka, Kagoshima 890-8520, Japan. ${ }^{2}$ Department of Pathology, Tokai University School of Medicine, 143 Shimokasuya, Isehara, Kanagawa, Japan. ${ }^{3}$ Department of General Thoracic Surgery, Graduate School of Medical and Dental Sciences, Kagoshima University, 8-35-1 Sakuragaoka, Kagoshima, Japan.

Received: 17 May 2017 Accepted: 29 November 2017

Published online: 12 December 2017

\section{References}

1. World Health Organization. Cancer. Fact Sheet Number 297. www.who. int/mediacentre/factsheets/fs297/en/ Last updated February 2015. Accessed 10 Feb 2015

2. Kennedy GT, Okusanya OT, Keating JJ, Heitjan DF, Deshpande C, Litzky LA, Albelda SM, Drebin JA, Nie S, Low PS, et al. The optical biopsy: a novel technique for rapid Intraoperative diagnosis of primary pulmonary Adenocarcinomas. Ann Surg. 2015;262(4):602-9.

3. Lim HJ, Ahn S, Lee KS, Han J, Shim YM, Woo S, Kim JH, Yie M, Lee HY, Yi CA Persistent pure ground-glass opacity lung nodules $>/=10 \mathrm{~mm}$ in diameter at CT scan: histopathologic comparisons and prognostic implications. Chest. 2013;144(4):1291-9.

4. Slatore CG, Horeweg N, Jett JR, Midthun DE, Powell CA, Wiener RS, Wisnivesky JP, Gould MK. An official American Thoracic Society research 
statement: a research framework for pulmonary nodule evaluation and management. Am J Respir Crit Care Med. 2015;192(4):500-14.

5. Okada M, Nishio W, Sakamoto T, Uchino K, Yuki T, Nakagawa A, Tsubota N. Prognostic significance of perioperative serum carcinoembryonic antigen in non-small cell lung cancer: analysis of 1,000 consecutive resections for clinical stage I disease. Ann Thorac Surg. 2004;78(1):216-21.

6. Zenita K, Kirihata Y, Kitahara A, Shigeta K, Higuchi K, Hirashima K, Murachi T, Miyake M, Takeda T, Kannagi R. Fucosylated type-2 chain polylactosamine antigens in human lung cancer. Int J Cancer. 1988;41(3):344-9.

7. Satoh H, Ishikawa H, Kamma H, Yamashita YT, Takahashi H, Ohtsuka M, Hasegawa S. Serum sialyl lewis X-i antigen levels in non-small cell lung cancer: correlation with distant metastasis and survival. Clin Cancer Res. 1997;3(4):495-9.

8. Rivera MP, Mehta AC, Wahidi MM. Establishing the diagnosis of lung cancer: diagnosis and management of lung cancer, 3rd ed: American College of Chest Physicians evidence-based clinical practice guidelines. Chest. 2013; 143(5 Suppl):e142S-65S.

9. Wiener RS, Wiener DC, Gould MK. Risks of transthoracic needle biopsy: how high? Clin Pulm Med. 2013;20(1):29-35.

10. Wiener RS, Schwartz LM, Woloshin S, Welch HG. Population-based risk for complications after transthoracic needle lung biopsy of a pulmonary nodule: an analysis of discharge records. Ann Intern Med. 2011;155(3):137-44.

11. Watanabe M, Ishizaka A, Ikeda E, Ohashi A, Kobayashi K. Contributions of bronchoscopic microsampling in the supplemental diagnosis of small peripheral lung carcinoma. Ann Thorac Surg. 2003;76(5):1668-72. discussion 1673

12. Ishizaka A, Watanabe M, Yamashita T, Ogawa Y, Koh H, Hasegawa N, Nakamura H, Asano K, Yamaguchi K, Kotani M, et al. New bronchoscopic microsample probe to measure the biochemical constituents in epithelial lining fluid of patients with acute respiratory distress syndrome. Crit Care Med. 2001:29(4):896-8.

13. Ishizaka A, Matsuda T, Albertine KH, Koh H, Tasaka S, Hasegawa N, Kohno N, Kotani T, Morisaki H, Takeda J, et al. Elevation of KL-6, a lung epithelial cell marker, in plasma and epithelial lining fluid in acute respiratory distress syndrome. Am J Physiol Lung Cell Mol Physiol. 2004;286(6):L1088-94.

14. Koh H, Tasaka S, Hasegawa N, Asano K, Kotani T, Morisaki H, Takeda J, Fujishima S, Matsuda T, Hashimoto S, et al. Vascular endothelial growth factor in epithelial lining fluid of patients with acute respiratory distress syndrome. Respirology. 2008;13(2):281-4.

15. Kamiya K, Watanabe M, Kohno M, Izumi Y, Horinouchi H, Kawamura M, Shimada N, Nomori H. KL-6 and CEA levels in epithelial lining fluid microsamples predict response to gefitinib in patients with advanced nonsmall cell lung cancer. Respirology. 2011;16(6):976-82.

16. Kipnis E, Hansen K, Sawa T, Moriyama K, Zurawel A, Ishizaka A, WienerKronish J. Proteomic analysis of undiluted lung epithelial lining fluid. Chest. 2008;134(2):338-45.

17. Kahn N, Kuner R, Eberhardt R, Meister M, Muley T, Winteroll S, Schnabel PA, Ishizaka A, Herth FJ, Poustka A, et al. Gene expression analysis of endobronchial epithelial lining fluid in the evaluation of indeterminate pulmonary nodules. J Thorac Cardiovasc Surg. 2009;138(2):474-9.

18. Yasuda H, Soejima K, Nakayama S, Kawada I, Nakachi I, Yoda S, Satomi R, Ikemura S, Terai H, Sato T, et al. Bronchoscopic microsampling is a usefu complementary diagnostic tool for detecting lung cancer. Lung Cancer. 2011;72(1):32-8

19. Kahn N, Meister M, Eberhardt R, Muley T, Schnabel PA, Bender C, Johannes M, Keitel D, Sultmann $H$, Herth FJ, et al. Early detection of lung cancer by molecular markers in endobronchial epithelial-lining fluid. J Thorac Oncol. 2012;7(6):1001-8

20. Brasch F, Ochs M, Kahne T, Guttentag S, Schauer-Vukasinovic V, Derrick M, Johnen G, Kapp N, Muller KM, Richter J, et al. Involvement of napsin A in the $\mathrm{C}$ - and $\mathrm{N}$-terminal processing of surfactant protein B in type-II pneumocytes of the human lung. J Biol Chem. 2003;278(49):49006-14.

21. Ueno $T$, Linder $\mathrm{S}, \mathrm{Na} C L$, Rice $W R$, Johansson J, Weaver TE. Processing of pulmonary surfactant protein B by napsin and cathepsin $\mathrm{H}$. J Biol Chem. 2004:279(16):16178-84

22. Woischnik M, Bauer A, Aboutaam R, Pamir A, Stanzel F, de Blic J, Griese M. Cathepsin $\mathrm{H}$ and napsin $\mathrm{A}$ are active in the alveoli and increased in alveolar proteinosis. Eur Respir J. 2008;31(6):1197-204.

23. Ueno $\mathrm{T}$, Linder $\mathrm{S}$, Elmberger $\mathrm{G}$. Aspartic proteinase napsin is a useful marker for diagnosis of primary lung adenocarcinoma. Br J Cancer. 2003;88(8):1229-33.

24. Turner BM, Cagle PT, Sainz IM, Fukuoka J, Shen SS, Jagirdar J. Napsin A, a new marker for lung adenocarcinoma, is complementary and more sensitive and specific than thyroid transcription factor 1 in the differential diagnosis of primary pulmonary carcinoma: evaluation of 1674 cases by tissue microarray. Arch Pathol Lab Med. 2012;136(2):163-71.

25. Kim MJ, Shin HC, Shin KC, Ro JY. Best immunohistochemical panel in distinguishing adenocarcinoma from squamous cell carcinoma of lung: tissue microarray assay in resected lung cancer specimens. Ann Diagn Pathol. 2013;17(1):85-90.

26. Wang Memoli JS, Nietert PJ, Silvestri GA. Meta-analysis of guided bronchoscopy for the evaluation of the pulmonary nodule. Chest. 2012; 142(2):385-93.

27. Oki M, Saka H, Ando M, Asano F, Kurimoto N, Morita K, Kitagawa C, Kogure Y, Miyazawa T. Ultrathin Bronchoscopy with multimodal devices for peripheral pulmonary lesions. A randomized trial. Am J Respir Crit Care Med. 2015;192(4):468-76.

28. Mondoni M, Sotgiu G, Bonifazi M, Dore S, Parazzini EM, Carlucci P, Gasparini S, Centanni S. Transbronchial needle aspiration in peripheral pulmonary lesions: a systematic review and meta-analysis. Eur Respir J. 2016;48(1):196-204.

29. Sharma R, Wang Y, Chen L, Gurda GT, Geddes S, Gabrielson E, Askin F, Li QK. Utility of a novel triple marker (combination of TTF-1, Napsin-A and P40) in the subclassification of non-small cell lung carcinomas using fine needle aspiration cases. Hum Pathol. 2016;54:8-16.

30. Hirano T, Gong Y, Yoshida K, Kato Y, Yashima K, Maeda M, Nakagawa A Fujioka K, Ohira T, Ikeda N, et al. Usefulness of TA02 (napsin A) to distinguish primary lung adenocarcinoma from metastatic lung adenocarcinoma. Lung Cancer. 2003;41(2):155-62.

31. Mitchell KF. A Carcinoembryonic antigen (CEA) specific monoclonal hybridma antibody that reacts only with high-molecular-weight CEA. Cancer Immunol Immunother. 1980;10:1-5.

32. Samukawa T, Hamada T, Uto H, Yanagi M, Tsukuya G, Nosaki T, Maeda M, Hirano $T$, Tsubouchi $H$, Inoue $H$. The elevation of serum napsin A in idiopathic pulmonary fibrosis, compared with $\mathrm{KL}-6$, surfactant protein-a and surfactant protein-D. BMC Pulm Med. 2012;12:55

33. Schneider J. Tumor markers in detection of lung cancer. Adv Clin Chem 2006:42:1-41.

34. Lai RS, Hsu HK, Lu JY, Ger LP, Lai NS. CYFRA 21-1 enzyme-linked immunosorbent assay. Evaluation as a tumor marker in non-small cell lung cancer. Chest. 1996:109(4):995-1000.

35. Grunnet M, Sorensen JB. Carcinoembryonic antigen (CEA) as tumor marker in lung cancer. Lung Cancer. 2012;76(2):138-43.

\section{Submit your next manuscript to BioMed Central and we will help you at every step:}

- We accept pre-submission inquiries

- Our selector tool helps you to find the most relevant journal

- We provide round the clock customer support

- Convenient online submission

- Thorough peer review

- Inclusion in PubMed and all major indexing services

- Maximum visibility for your research

Submit your manuscript at www.biomedcentral.com/submit

Biomed Central 